\title{
A Novel q-parameter Automation in Tsallis Entropy for Image Segmentation
}

\author{
M Seetharama Prasad \\ KL University \\ Vijayawada- 522202
}

\author{
P Radha Krishna \\ KL University \\ Vijayawada- 522202
}

\begin{abstract}
Image Thresholding is the necessary task in some image processing applications. The goal of any segmentation technique in image processing is to find out the object from the background in the given image. In this paper, q-parameter of the Tsallis entropy is analyzed in the existing and the proposed methods. Different techniques have been introduced to automate the q-parameter to obtain corresponding threshold value. After the comparison of all results by using misclassification error, the proposed one yields better results as demonstrated by statistical analysis.
\end{abstract}

\section{Key words}

Tsallis entropy, Image Thresholding, q-parameter.

\section{INTRODUCTION}

Image Thresholding is a necessary task in some image processing applications. Segmentation subdivides an image into regions or objects. Convert an image into a representation that is more meaningful and easier to analyze. It assigns a label to every pixel such that pixels with the same label share certain visual characteristics. Thresholding is the partition images directly into regions based on intensity values and/or properties of these values. Image analysis usually refers to processing of images with the goal of finding objects presented in the image. Image segmentation is one of the most critical tasks in automatic image analysis. Image Thresholding is used to divides the image in to foreground and object. The gray level image is converting the binary level image by using Thresholding techniques. Accuracy of segmentation is depends upon the process which is based on the gray level histogram. From the literature pun[1] that first applied the concept of entropy to Thresholding. His methods concludes when the sum of the background and object entropies reaches its maximum, the image threshold is obtained. Kapur et al.[2] propose a method based on the previous work of pun[1]in which, Images which are corrupted with noise or irregular illumination produce multimodal histograms in which a 2D histogram does not guarantee the optimum introduced a new image segmentation procedure based on entropy. In this paper we used the Tsallis entropy proposed the different types of Thresholding techniques. Abutaleb[4] introduced a novel idea considering the spatial correlation among the gray pixels in the image. Yang Zio et. al [5] modified this idea as Abutaleb's method, convergence is so late. Seetharama Prasad et. al [6] further modified reference [5] by introducing by modifying the dynamic similarity measure.

The organization of this paper is as follows, The introduction part is presented in section 1 followed by Section 2 describes the Existing method of Tsallis entropy. Section 3 is dedicated to the three Proposed methods to automate the qparameter in the existing formula of Tsallis entropy. Section 4 demonstrates all the results and their analysis with misclassification error. Conclusions are explained in Section5 and as usual references are given in Section 6.

\section{EXISTING METHOD}

For an image with $\mathrm{k}$ gray-levels, let $p_{i}=p_{1}, p_{2}, \ldots \ldots p_{k}$. be the probability distribution of the levels. From this distribution two probability distributions are derived, one for the object (class A) and another for the background (class B). The probability distributions of the object and background classes, $\mathrm{A}$ and $\mathrm{B}$, are given by

$P_{A}=\frac{p_{1}}{p^{A}}, \frac{p_{2}}{p^{A}}, \ldots \ldots \ldots \ldots \frac{p_{t}}{p^{A}}$
$P_{B}=\frac{p_{t+1}}{p^{B}}, \frac{p_{t+2}}{p^{B}}, \ldots \ldots \ldots \ldots \frac{p_{k}}{p^{B}}$

Where $p^{A}=\sum_{i=1}^{t} p_{i}$ and $p^{B}=\sum_{i=t+1}^{k} p_{i}$

The a priori Tsallis entropy for each distribution is defined as

$S_{q}^{A}(\mathrm{t})=\frac{1-\sum_{i=1}^{t}\left(\frac{p_{i}}{p^{A}}\right)^{q}}{q-1}$

$S_{q}^{B}(\mathrm{t})=\frac{1-\sum_{i=t+1}^{k}\left(\frac{p_{i}}{p^{B}}\right)^{q}}{q-1}$

The Tsallis entropy $S_{q}^{A}(\mathrm{t}), S_{q}^{B}(\mathrm{t})$ is parametrically dependent upon the threshold value $\mathrm{t}$ for the foreground and background. It is formulated as the sum of each entropy, allowing the pseudo-additive property for statistically independent systems, defined

$S_{t}(\mathrm{t})=\frac{1-\sum_{i=1}^{t}\left(\frac{p_{i}}{p^{A}}\right)^{q}}{q-1}+\frac{1-\sum_{i=t+1}^{k}\left(\frac{p_{i}}{p^{B}}\right)^{q}}{q-1}+(1-\mathrm{q})^{*} \frac{1-\sum_{i=1}^{t}\left(\frac{p_{i}}{p^{A}}\right)^{q}}{q-1}$
$* \frac{1-\sum_{i=t+1}^{k}\left(\frac{p_{i}}{p^{B}}\right)^{q}}{q-1}$ the information measure between the two classes (object and background) has been maximized. When $s_{t}(\mathrm{t})$ is maximized, 
the luminance level $\mathrm{t}$ is considered to be the optimum threshold value. This can be achieved with a cheap computational effort.

$t_{\text {opt }}=\operatorname{argmax}\left(S_{q}^{A}(\mathrm{t})+S_{q}^{B}(\mathrm{t})+(1-\mathrm{q}) * S_{q}^{A}(\mathrm{t}) * S_{q}^{B}(\mathrm{t})\right)$

It is totally supervised method, in every time to check the result need to change the parameter $\mathrm{q}$ and threshold value $\mathrm{t}$. In this paper $\mathrm{q}=1.0$, and $\mathrm{t}=127$ is taken in the existing method.

\section{PROPOSED METHODS}

\subsection{Method 1}

In this method parameter $\mathrm{q}$ is taken the mean value of the image and threshold value $\mathrm{t}$ is fixed 127. Remaining procedure is same as Tsallis entropy. As the last step of the algorithm optimal threshold value is derived, the gray value for which the maximum entropy is obtained.

\subsubsection{Algorithm}

1. Read the image, if the images contrast less than $90^{\circ}$ then multiplies the 2 .

2. Calculate the mean value of the image, it is assign to $\mathrm{q}$, and take the threshold value $\mathrm{t}=127$ it is fixed.

3. Calculate the image histogram.

4. Calculate the probability of each pixel.

5. Calculate the Tsallis entropy when the maximum value is occurred then it locate the optimal value $=\mathrm{T}$.

$$
\text { Mean } \mu=\frac{1}{N} \sum_{1}^{n} x_{i} * h_{i}
$$

\subsection{Method 2}

In this method $\mathrm{q}$ and $\mathrm{t}$ value is taken the mean value of the image it is totally supervised method remaining procedure as same as Tsallis entropy. At lost to find out the optimal threshold value.

\subsubsection{Algorithm}

1. Read the image, if the images contrast less than $90^{\circ}$ then multiplies the 2 .

2. Calculate the mean value of the image, it is assign to $\mathrm{q}$ and $\mathrm{t}$

3. Calculate the image histogram.

4. Calculate the probability of each pixel.

5. Calculate the Tsallis entropy when the maximum value is occurred then it locate the optimal value $=\mathrm{T}$.

\subsection{Method 3}

In this method, the parameter ' $\mathrm{q}$ ' is assigned the image standard deviation value, and the mean value of the image is assign to threshold value ' $t$ '. The probability of each pixel in the image is estimated. For an image with $\mathrm{k}$ gray-levels, let $p_{i}=p_{1}, p_{2}, \ldots \ldots p_{k}$. be the probability distribution of the levels. From this distribution two probability distributions have been derived, one for the object and another for the background. The probability distributions of the object and background classes, $\mathrm{A}$ and $\mathrm{O}$, are given by

$P_{A}=\frac{p_{1}}{p^{A}}, \frac{p_{2}}{p^{A}}, \ldots \ldots \ldots \ldots . . \frac{p_{t}}{p^{A}}$

$P_{B}=\frac{p_{t+1}}{p^{B}}, \frac{p_{t+2}}{p^{B}}, \ldots \ldots \ldots \ldots \frac{p_{k}}{p^{B}}$

Where $p^{A}=\sum_{i=1}^{t} p_{i}$ and $p^{B}=\sum_{i=t+1}^{k} p_{i}$

$P_{b}=\left(P_{A}\right)^{q}$

$P_{o}=\left(P_{B}\right)^{q}$

$P_{b}$ is the probability of the background image the optimal threshold value $T_{1}$ is taken from the maximum value locate the position in the background image. $P_{o}$ is the probability of the object image the optimal threshold value $T_{2}$ is taken from the maximum value locate the position in the object image. The optimal value $\mathrm{T}$ is taken from the

$$
\mathrm{T}=\frac{T_{1}+\frac{T_{2}}{2}}{2}
$$

\subsubsection{Algorithm}

1. Read the image, if the images contrast less than $90^{\circ}$ then multiplies the 2

2. Find out the standard deviation and mean of the image and the value is assign to $\mathrm{q}$ and threshold value $t$ respectively.

3. Calculate the image histogram

4. Calculate the $P_{B}$ and $P_{O}$ (from the above equations).

5. Find out the maximum values $P_{B}$ and $P_{O}$ locate the positions and to assign the optimal value $T_{1}$ and $T_{2}$ subsequently.

6. Find out the optimal threshold value $\mathrm{T}=\frac{T_{1}+\frac{T_{2}}{2}}{2}$.

Mean $\mu=\frac{1}{N} \sum_{1}^{n} x_{i} * h_{i}$

Standard deviation $\sigma=\sqrt{\frac{1}{N} \sum_{i}^{n} x_{i}\left(x_{i}-\mu\right)^{2}}$

\section{RESULTS AND DISCUSSIONS}

To illustrate the performance of the proposed methodology 15 images have been considered as an image set having similar and dissimilar gray level histogram characteristics, varying from uni-model to multimodal. Gold standard ground truth images are generated manually to measure parameter efficiency $(\eta)$ based on misclassification error[7].

\subsection{Misclassification Error}

Misclassification error $(\eta)=\frac{\left|I M G_{O} \cap I M G_{T}\right|}{I M G_{O}} * 100$

Where, IMGO, IMGT are gold standard image and resultant image respectively and $|*|$ is the Cartesian Number of the set gives number of pixels. This $\eta$ would be 0 for absolutely dissimilar and 100 for exactly similar image as result. Figure 1 shows proposed image set and their corresponding binary images of different methods along with ground truth images. 
Original Ground truth Existing Method1 Method2 Proposed
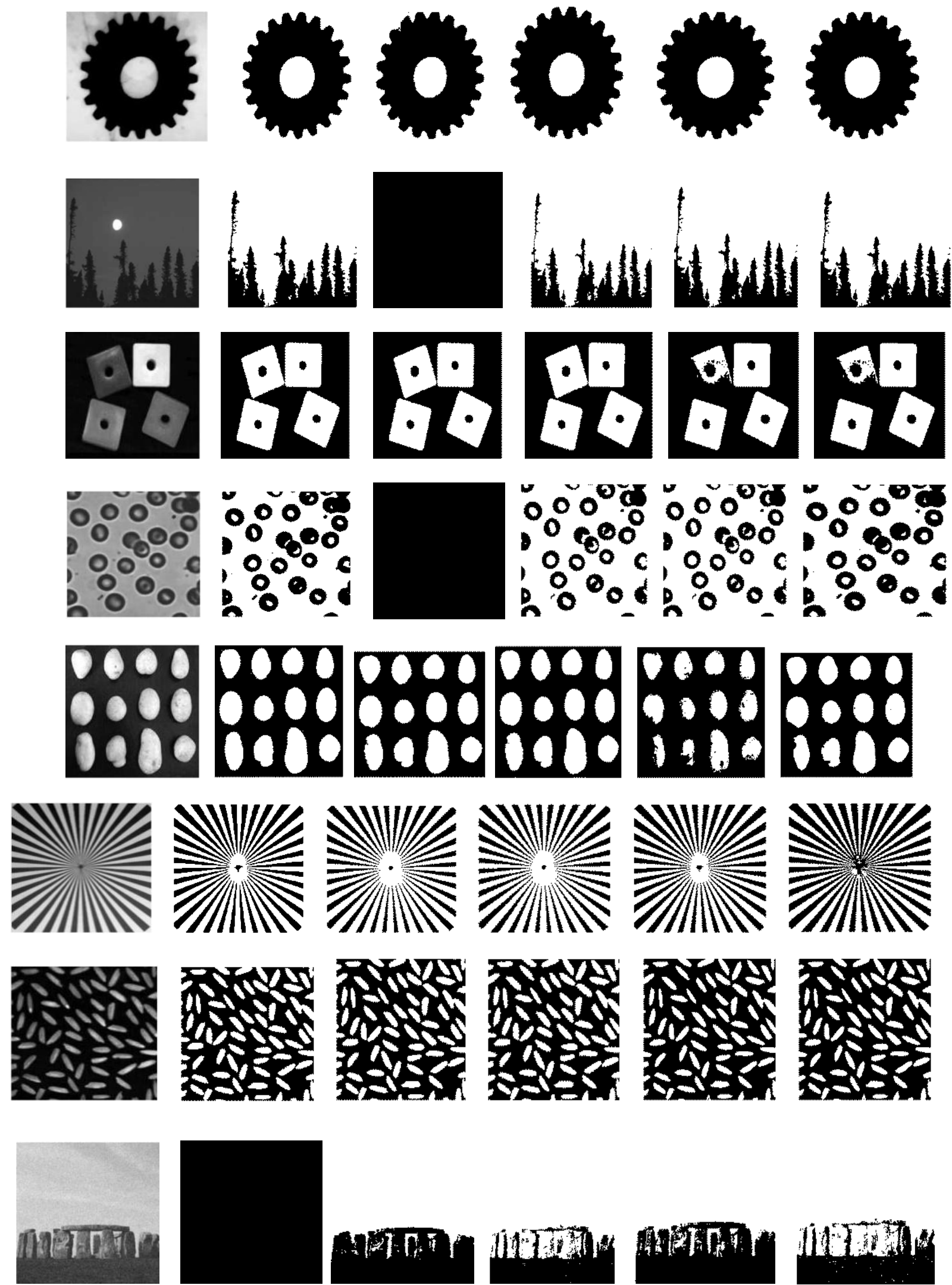

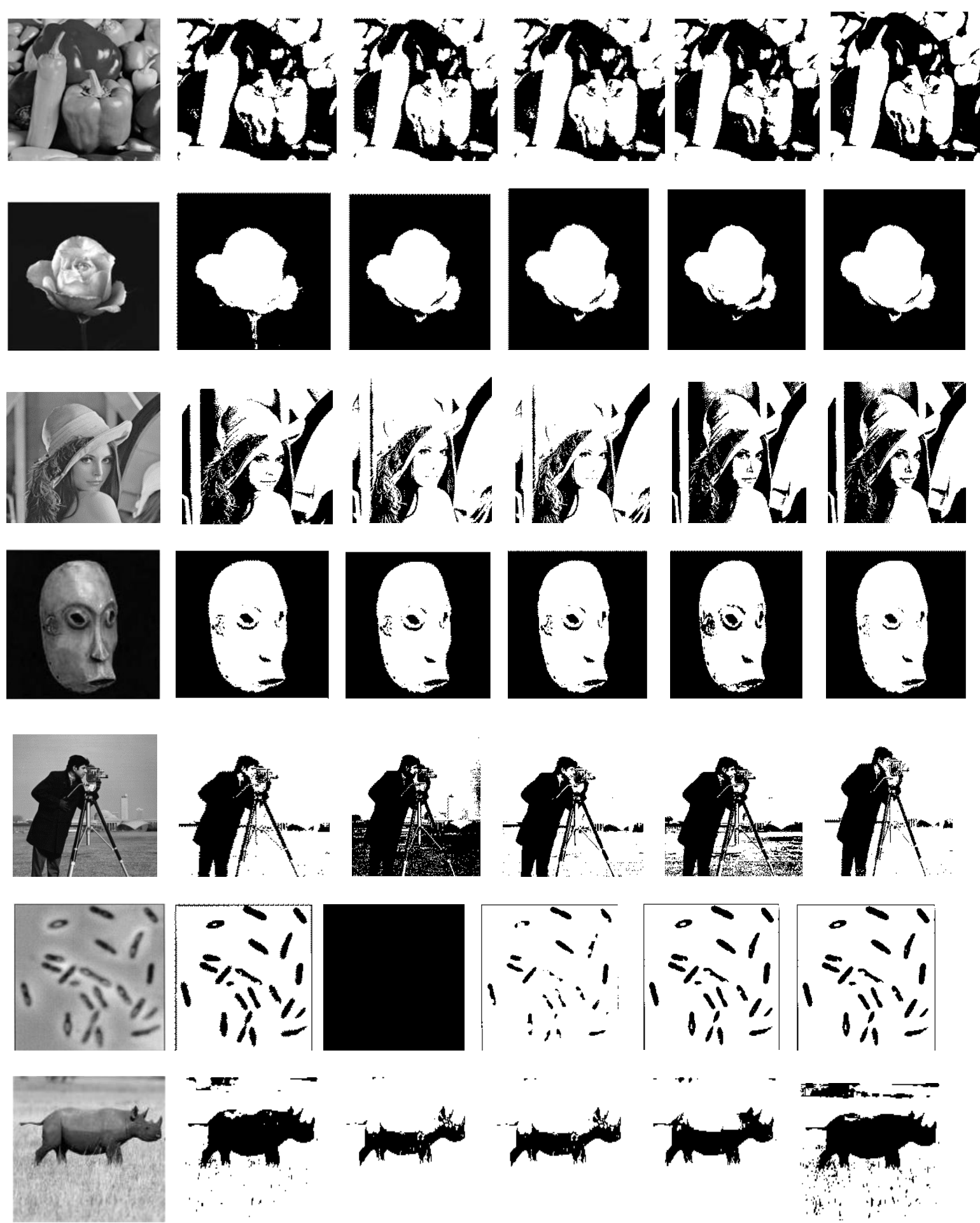

Fig 1: (From left to right) Data set, ground truth images and corresponding results for the algorithms Tsallis, method1, method2 and Proposed. 
Table 1: Efficiency using Misclassification Error ( $\eta$ \%)

\begin{tabular}{|c|l|c|c|c|c|}
\hline Sl. no & Image name & Tsallis & Method 1 & Method 2 & Proposed \\
\hline 1 & Wheel & 97.39 & 99.2 & 99.2 & 99.73 \\
\hline 2 & Trees & 25.57 & 97.59 & 97.59 & 99.80 \\
\hline 3 & Blocks & 100 & 100 & 100 & 94.81 \\
\hline 4 & Blood cells & 30.49 & 95.35 & 95.35 & 99.60 \\
\hline 5 & Potatoes & 99.04 & 99.04 & 99.04 & 96.54 \\
\hline 6 & ohm & 94.42 & 94.42 & 94.42 & 93.60 \\
\hline 7 & Rice & 100 & 100 & 100 & 95.68 \\
\hline 8 & Stones & 35.41 & 90.32 & 90.32 & 89.48 \\
\hline 9 & Pepper & 96.55 & 96.55 & 96.55 & 99.37 \\
\hline 10 & Rose & 99.01 & 99.01 & 97.72 & 99.08 \\
\hline 11 & Lena & 82.66 & 82.66 & 95.32 & 93.63 \\
\hline 12 & Zimba & 99.81 & 99.81 & 99.81 & 99.56 \\
\hline 13 & Cameraman & 74.65 & 99.24 & 99.21 & 99.37 \\
\hline 14 & Bacteria & 13.84 & 92.33 & 92.33 & 97.48 \\
\hline 15 & Rhino & 88.25 & 88.25 & 88.25 & 94.51 \\
\hline & Mean $(\mu)$ & 74.26 & 95.32 & 96.14 & 96.61 \\
\hline
\end{tabular}

From the experiments for each image misclassification error $(\boldsymbol{\eta} \%)$ is obtained for Tsallis and proposed methods as shown in TABLE 1. These values are compared with assumed gold standard image data. Figure 2 confirms a variation in above said methods on histogram range for image set considered against Tsallis method. Efficiency $(\eta)$ is calculated for each technique on image set with mean Equation. A mean $(\mu)$ calculated on efficiency in order to show the effectiveness of the proposed and other methods as in TABLE 1. A mean quantities of $95.32,96.13$ and 96.61 is obtained from the three proposed methods which confirms the quantitative improvement over the existing method.

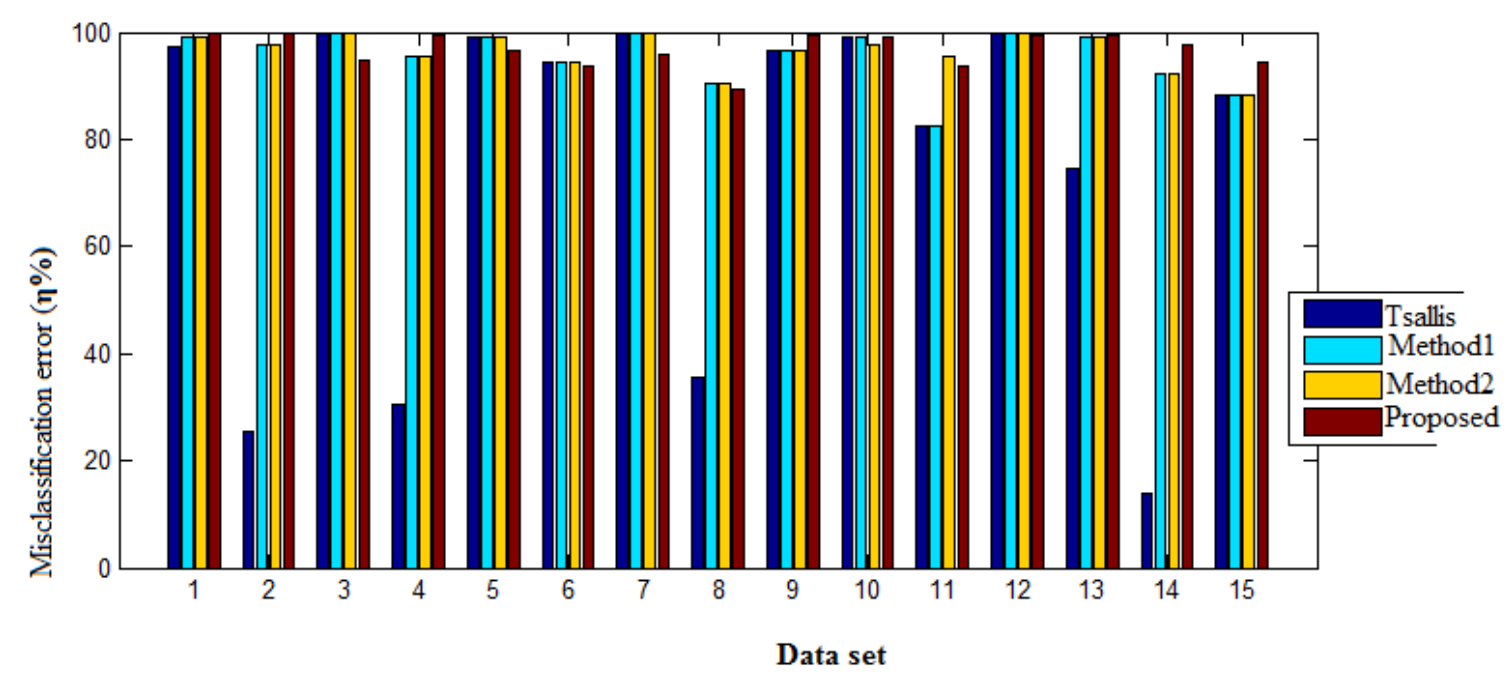

Fig: 2 Efficiency comparison of the proposed methods against Tsallis using Misclassification error

\section{CONCLUSIONS}

In this paper an automated segmentation approach based on Tsallis entropy method. The existing method the parameter ' $q$ ' and threshold value which not very easy to converge. In this paper automation of the ' $\mathrm{q}$ ' parameter is tried to obtain the threshold value. Three different methods are proposed and tested but the last method yields better results than others. Efficiency of threshold selection is demonstrated with experimental results. There is still lot of scope to consider the image other parameters like image vagueness; and fuzziness, by employing fuzzy image segmentation techniques in combination with Tsallis entropy procedure this can be further improvised. A reasonable contrast enhancement is assumed for low contrast images. Performance evolution is carried out with the help of Misclassification error on the proposed work. 


\section{REFERENCES}

[1] Pun, T., 1981. Entropic thresholding: A new approach. Comput. Graphics Image Process. 16, 210-239.

[2] Kapur, J.N., Sahoo, P.K., Wong, A.K.C., 1985. A new method for gray-level picture thresholding using the entropy of the histogram. Comput. Vision Graphics Image Process. 29, 273-285.

[3] Tsallis, 2004. Image thresholding using Tsallis entropy. Pattern Recognition Letters 25 (2004) 1059-1065

[4] Abutaleb, A.S., 1989. Automatic thresholding of gray level pictures using two dimensional entropy. Comput. Vision Graphics Image Process. 47, 22-32.
[5] Y.Xiao, Z.G.Cao, and S.Zhong, "New entropic thresholding approach using gray-level spatial correlation histogram", Optics Engineering, 49, 127007, 2010

[6] M Seetharama Prasad, T Divakar, L S S Reddy, "Improved Entropic Thresholding based on GLSC histogram with varying similarity measure", International Journal of Computer Applications, vol.24, June 2011.

[7] M. Sezgin and B. Sankur, "Survey over image thresholding techniques and quantitative performance evaluation," J. Electron. Imag., vol. 13, no. 1, pp. 146165, Jan. 2004 